ture, one alone of these being non-malignant. In all, the obstruction was almost complete, and the patients were reduced to the last stage of exhaustion by starvation before the operation was proposed; and here we find the chief causes of failure-pre-existing blood-change, and inability to resist shock. In the latter stages of malignant growth, it is obvious, moreover, that the blood has undergone other changes than the mere withdrawal of its watery elements: there is distinct dyscrasia, and the blood is not in a fit state to serve the purposes of nutrition and reparation; and this want oî reparative power is fully as formidable as the mere exhaustion incidental to previous starvation, or even more so: the one may, the other cannot, be overcome.

These are the most important conditions, it seems to me, in producing failure, so far as the patient is concerned. What are the causes of failure quoad the operation? There does not appear any reason to suppose that the mere incision into the stomach is by any means an extremely hazardous operation. The case of Alexis St. Martin shows well how reparation may take place under far more severe injury to that organ. So with Dr. Murchison's case. But the celebrated case of Shoral's proves that the operation in itself may be perfectly successful. In what, then, does the operation of Shoral differ? Simply in the fact that the wound in the stomach was immediately closed; that there was no strain upon it, and no tension of the nerves supplying it. The union of the stomach to the wound is apt to give rise to cough, and, when food is introduced, to efforts at vomiting. Both these conditions are extremely hazardous, and tend greatly to render the operation fatal by retarding the progress of adhesion, and even, as in Sédillot's case, tearing out the sutures.

Cough appeared in two of the six cases; vomiting* in two; and in two (Fenger's and Jones's) they are not mentioned. The apparent cause of death was, in four of the cases, exbaustion; in one, embolism ; and in one, peritonitis. Peritonitis appeared in two cases only-namely, in Sédillot's and in Mr. Forster's second case. In the former it arose from vomiting, which produced separation of the stitches and withdrawal of the stomach from the wound; in the latter, from separation of the adhesions through the introduction of a tube into the stomach.

The age of the patient is not an unimportant item in enumerating the causes of failure. Five out of the six were over the age of forty-four, and three of these were over fifty. The remaining one was four years old; and, looking at this case independently of the unfortunate accident above-mentioned, one would say that it ought to have proved successful.

What precautions could have obviated the fatal result in all these cases? Clearly, we may gather this from their consideration, that if an operation in any similar instance is to have any chance of success, it must be done at an earlier period, while there is still some power of reparation left, and at a time when there is yet some material to draw upon, so that we are not compelled to put food into the stomach at an inopportune moment, when it ought to be at rest; for after the operation it seems imperative that the stomach should be kept empty and the patient supported by nutritive enemata. We should thus avoid the great risk of vomiting, which is almost necessarily fatal, since the food cannot return by the closed œsophagus, and must therefore remain as a continual excitant of expulsive effort. The introduction of the tube ought always to be effected in the first instance, as it is difficult and, as Mr. Forster's case proves, hazardous afterwards. The point at which the stomach should be opened ought to be one as far removed as possible from a fixed point, so as to cause as little tension as may be. Mr. Jones found considerable difficulty, in his case, in fixing the stomach, owing to the fact of his having seized it near the cardiac end. The closure of the wound with colloid styptic is, I think, a great advantage; and it is important to limit the action of the abdominal walls by means of strapping and bandage, so as to keep the wound as much as possible in a state of rest.

With these brief and imperfect considerations, I would, in conclusion, ask, Is the operation a justifiable one? If the cases already recorded be all we have to judge by, the answer is obvious. The data, it is true, are limited, but

* Vomiting appears as a prominent cause of death in several cases of colotomy, and was at ribuled to the chloroform. Mr. J. Couper's case, for instance, eshibited no uther assignable cause (Med. Jour., Nov. 20ih, 1869). also Mr. Miaunder's and Mr. Curling's (Dis. of Test., 3rd ed., p. 177). they are wonderfully cogent. 'To answer such a question we cannot, however, be satisfied with so narrow a basis of argument. The real question is, not whether an operation is justifiable when performed under the same conditions as these, but whether it is justifiable under any conditions. When, for instance, the disease is of limited extent, the state of the patient not one of actual starvation, the strength not yet exhausted, does the operation afford any chance of prolonging life? It may be said, and with much truth, that under these circumstances the patient would refuse to undergo the operation; but, if it is to succeed at all, it must certainly be done at this time. Most surgical authorities urge against the operation, that " the disease will certainly progress, and the operation cannot greatly prolong the life of the patient." But is not this equally true of Amussat's operation, and of others undertaken for the removal of malignant growths? What valid reason is there in favour of the one which does not exist for the other? Mainly, I apprehend, that malignant disease in the rectum requires earlier operaticn; and that liquid food can be swallowed when the obstruction is really greater and the disease more advanced than that of a cancerous bowel which has to give passage to solid matter. Is there any reason why gastrotomy should be less successful than colotomy if performed at the same stage of the disease? The same amount of relief would be afforded if the operation succeeded; the diseased parts would be at rest, and consequently the disease would make slower progress; the patient would be freed from the pangs of unsatisfied hunger; his last days made more supportable, and possibly his life considerably lengthened.

\section{ON THE MOVEMENT OF MUCUS IN THE TRACHEA AND LARYNX.}

\section{BY HENRY VEALE, M.D., STAFF SURGEON, BOMBAY.}

THE following observations regarding the movement of mucus in the trachea and larynx may perhaps be of interest to some members of the profession.

A gentleman in fair health had been troubled for several weeks with some huskiness of voice, a frequent desire when speaking to clear his throat by "hemming" or hawking, and occasional expectoration of a little viscid mucus. This was especially the case on his rising in the morning, when he used generally to expectorate one or two pellets of mucus, of about the size of a pea or a little larger, and latterly these had been slightly streaked or tinged with blood. He had no cough, strictly speaking; nor had he the least pain or difficulty of breathing.

It was at first thought that the complaint was confined to the back of the throat; but, after repeated examinations with the laryngoscope, it was ascertained that the mucus was not secreted by the fauces or larynx, but that it came from even below the bifurcation of the trachea; and that the pellets, as eventually expectorated, were formed in the following manner :-From the opening of one of the bronchi small bead-like particles of mucus wonld be seen to emerge from time to time, and then to ascend, generally either across the cartilaginous rings or along the back of the trachea; but sometimes, instead of passing directly upwards, they would travel partly round the tube in the grooves between the rings. Sometimes also they would come across and coalesce with other drops of mucus; and in this way a small pellet, perhaps of the size of a buckshot, would reach the upper part of the trachea. Here their movement would cease to be immediately upward, and become almost exclusively spiral-the direction being from right to left, as shown in the following diagram.

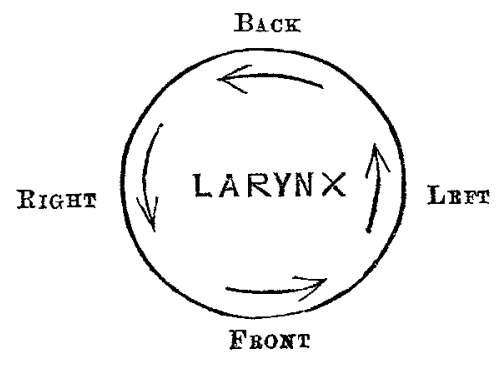


In the course of these circular or spiral movements in the larynx, the pellets could be seen to overtake one another and unite, until at length the mass so formed would reach the level of the vocal cords, and be expelled by a "hem" or a slight cough.

The rate at which the particles of mucus travelled round the windpipe varied. I never saw them make an entire circuit of the larynx in less than five minutes-usually they took from seven to eight minutes; and it is my impression that they moved more rapidly across the back of the larynx than in front or along either side, and much more rapidly round the larynx than round the trachea.

I have already mentioned that the mucus was occasionally tinged or streaked with blood, and this circumstance enabled me to identify the pellets in their change of place, and even after their expectoration. In the lower part of the trachea the circular movement is, I believe, quite exceptional, but I bave seen it occur in the left bronchus. The directly upward progression of the particles is always accelerated by the acts of phonation, and especially by those of deglutition, but I have not observed such acts to increase the rate at which the sputa travel round the larynx.

Voluntary efforts of coughing seem to have scarcely any power of expelling small quantities of mucus from the trachea, or even from the larynx, unless the particles are situated either immediately beneath the epiglottis, or just below and between the arytenoid cartilages. I have frequently found the most forcible acts of coughing that could be made voluntarily to fail in expelling a small pellet from the recess beneath a vocal cord. I have seen small particles of mucus adhering occasionally to the edge of a vocal cord; still, the back of the larynx is, I believe, the part from which such particles are most easily expectorated.

The huskiness of voice in the case which enabled me to make these observations appeared to be occasioned by the frequent arrival at the glottis of small particles of mucus. During the daytime these were expelled separately by the various "hems" and clearings of the throat which their presence at the glottis induced; but at night, during sleep, when the movements of the windpipe in speaking or swallowing were suspended, the particles had time to accumulate, and in this way the pellets were formed which were expectorated in the morning.

I should add that I have not perceived the spiral movement of mucus in the air-passages to be occasioned or accompanied by any movement of the mucous membrane, or any muscular action; but the particles often undergo a change of form in making the circuit. In the grooves between the rings, for instance, they are elongated; but on reaching the back of the tube they become flattened, and sometimes assume the appearance of a highly magnified amcba.

Bombay, April, 1871.

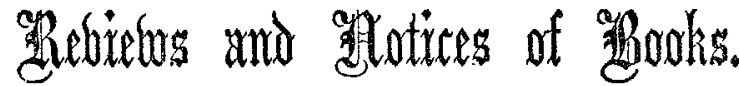

The Principles and Practice of Midwifery, with some of the Diseases of Women. By Alexander Milne, M.D. Illustrated with numerous Wood Engravings. Edinburgh: F. \& S. Livingstone. London: Simpkin, Marshall, and Co. 1871 .

Dr. MILne is an original and lively writer. The work is, of course, mainly obstetric in its subject; but it abounds throughout in lightsome digressions into the region of criticism, of pleasantry, of biography and autobiography, and even in exercises in versification, which are certainly of a most extraordinary character, and of the order met with in valentines and in the mottoes found inside of crackers. Before one gets far in to the preface, these peculiarities of the author appear. He makes almost unnecessary opportunity to excuse his faults. "An imperfect sentence, a dull paragraph, or even a tedious chapter, may be often credited to a tedious labour, invading the period when the cerebrum should have indispensable repose." Then he goes on to cast the mantle of the same consideration over a busy physician in Edinburgh, well known to and much liked by us all, who " not long ago produced a highly creditable trans- lation of the Satires of Horace." Who can be hard upon such an author as this? The work contains a very fair account of the present state of the art and science of midwifery, adapted alike to students and practitioners. Frequently the lively and digressive style of the author annoys the reader by interfering with the even course of the subject, or by disturbing the grave and scientific state of mind; but, for all this, Dr. Milne has contrived to condense a great amount of obstetric doctrine, both physiological and pathological, into his book, and to criticise it practically in the light of anatomical and physiological facts and of a certain amount of practice in the art of midwifery and of its difficult parts. The book is at once theoretical and practical. The author displays a considerable knowledge of obstetric literature in all its branches, and, at the same time, speaks with the discrimination of a practitioner. The teaching concerning the early resort to instrumental assistance in suitable cases is in accordance with the best experience of obstetricians. There is an excellent account of cephalotripsy, spondylotomy, and other later obstetric operations, as of the different modes of inducing premature labour, treating uterine hæmorrhage, \&c.

The matter of the book is divided into no less than 73 chapters. This seems a formidable quantity. But the chapters are neither long nor exhausting, and it is difficult to find the author overlooking any subject or any material point of importance in connexion with it. Occasionally serious subjects are disposed of in an offhand and somewhat jaunty way; as, for example, that of the treatment of cellulitis. "There is reason to believe that an incipient cellulitis," we are told, "may be aborted by early antiphlogistic treatment." Leeches, a saline purgative, and one good dose of calomel (five to ten grains) at the outset, are the author's drugs, and his chief treatment. $\mathrm{He}$ is an unshaken believer in the antiphlogistic virtue of mercury. With a characteristic tendency to the careless use. of figurative language, he says, "for reducing granitic hardness no remedy can hold a candle to it." ......... He disposes. of objections to it in a very easy way, probably very different from the way in which he would speak if he were under the action of the remedy he is recommending. "If that dose. should salivate, it can't be helped; better fluid gushing from the mouth than cooped up in the belly; confined in a very important locality, and no saying when or where it may escape." It is true that it is recommended to follow the purgative up with an opiate, and that perfect rest is enjoined; but there is a careless endorsement of a very much discredited practice here, which is open to very serious objection. The same faith in old antiphlogistics, and "the worthies who toiled away" in the middle of epidemics of peritonitis in the beginning of the century, using profuse bleeding and no less liberal purgation, appears at various parts of the book, and seems to us to be unworthy of the generally modern character of it. The author will forgive us for advising him to lay aside his tendency to use figures, and to parenthetical pleasantries and personalities when treating of such grave subjects as four grains of calomel three times a day in puerperal peritonitis, even with opium. Barring this tendency to recommend bleeding and calomel, the therapeutics of the book are modern, and not injudicious; but, speaking generally, the paragraphs on treatment want to be rewritten with more gravity and care. Even those on the dietetic treatment of the puerperal female are written with a looseness that is liable to be seriously misunderstood, though the main intention of the author, judging from other expressions, is correct. Arguing against too thin a diet in childbed, he says, "It is one thing to keep pouring stimulating diet into a patient when inflammation or fever have set in, but quite another to act thus 